International Journal of Pure and Applied Mathematics

Volume 97 No. 1 2014, 99-109

ISSN: 1311-8080 (printed version); ISSN: 1314-3395 (on-line version)

url: http://www.ijpam.eu

doi: http://dx.doi.org/10.12732/ijpam.v97i1.10

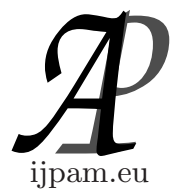

\title{
BRIEFLY REVISITING THE QUANTUM MECHANICS SUPERSYMMETRY ALGEBRA AND GROUP
}

\author{
R. Thibes $^{1 \S}$, E.F. Batista ${ }^{2}$, G.D. Barbosa ${ }^{3}$ \\ 1,2,3 Departamento de Ciências Exatas e Naturais \\ Universidade Estadual do Sudoeste da Bahia \\ Itapetinga, BA, BRASIL
}

\begin{abstract}
We review supersymmetry (SUSY) in nonrelativistic quantum mechanics emphasizing algebraic aspects. We discuss the Hamiltonian subgroup implementing supersymmetry as well as the corresponding algebra of the SUSY generators. In the SUSYQM framework, the two distinct partner potentials are connected by a superpotential satisfying a Riccati differential equation. A full Hamiltonian operator in an extended Hilbert space is defined in order to render the supersymmetry manifest. As a result, the eigenfunctions of the original potentials are connected by generalized ladder operators. We provide an explicit realization of the abstract supersymmetry group for SUSYQM depending on one real and two Grassmann parameters.
\end{abstract}

AMS Subject Classification: 08A99, 17B81, 81R15

Key Words: supersymmetry, supersymmetric quantum mechanics, factorization method, supergroups, superalgebra

\section{Introduction}

In a seminal paper analyzing the dynamical breaking of supersymmetry, published in 1981, Witten has proposed a simple model for supersymmetric quan-

Received: August 6, 2014

(C) 2014 Academic Publications, Ltd.

$\S$ Correspondence author url: www.acadpubl.eu 
tum mechanics (SUSYQM) [1]. In recent years, the role of supersymmetry (SUSY) in nonrelativistic quantum mechanics has been extensively analyzed, leading to a consistent classification of interacting potentials $[2,3,4,5]$. Firstly considered as an essential ingredient for any fundamental interactions unifying theory, SUSY has firmly established itself as an important mathematical technique for approaching problems, both in quantum mechanics and in quantum field theory, in its own right. Particularly, SUSYQM has been successfully used as a powerful method for analytically solving the Schrödinger equation for some potentials, as well as for constructing approximation methods for handling more involved not exactly solvable ones, establishing thus the eigenvalues and eigenvectors properties of the corresponding Schrödinger operators. Also in the relativistic case, SUSYQM has proved to be a suitable framework providing important insights to classical problems $[2,6,7]$. However, when it comes to realize the supersymmetry in terms of the action of an abstract group in the Hilbert space one faces some subtleties having to deal with supergroups and Grassmannian manifolds [8,9]. Although the SUSYQM algebra has been largely studied in the literature, little has been said about the symmetry group. We address this issue here obtaining explicitly the group law for one dimensional quantum mechanics implementing SUSYQM.

The main idea in SUSYQM is to relate the original Hamiltonian $H_{-}$for a certain problem to another one, $H_{+}$, known as the former's SUSY partner. This relation is achieved by means of introducing a superpotential $W(x)$ in the theory. SUSY connects the eigenfunctions of the two partner Hamiltonians in a simple way - the knowledge of one set of eigenfunctions permits one to directly calculate the other. Particularly, the SUSY partner potentials are isospectral. This leads to a symmetry (the supersymmetry) relating eigenfunctions of the distinct Hamiltonians $H_{-}$and $H_{+}$with the same energy. We shall show in the following that it is possible to define a new Hamiltonian operator comprising both $H_{-}$and $H_{+}$and realize the supersymmetry in a concrete way via the action of two nilpotent operators $Q^{ \pm}$. The invariance of this complete Hamiltonian under supersymmetry determines a realization of the corresponding supersymmetric group parametrized by real and Grassmann variables acting on the Hilbert space of quantum states.

Our work is organized as follows. In Section 2 below we review SUSYQM introducing the partner potentials $V_{ \pm}$and Hamiltonians $H_{ \pm}$, and establish their isospectrality. In Section 3 we construct the SUSYQM algebra acting in the full Hilbert space connecting the two original problems. The supercharge operators, together with the full Hamiltonian are shown to be the generators of a Lie superalgebra. Finally in Section 4 we construct the SUSYQM group of super- 
symmetry and calculate explicitly the group law in terms of three parameters. We conclude in Section 5 with some remarks.

\section{Supersymmetry in Quantum Mechanics}

Consider a spinless nonrelativistic particle subjected to a one dimensional timeindependent real potential $V(x)$. We are interested in the quantum mechanical description of bound states governed by the Schrödinger equation and assume $V(x)$ to be a confining potential. The well-known separation of variables technique can be applied leading to the time-independent Schrödinger equation

$$
-\frac{\hbar^{2}}{2 m} \frac{d^{2}}{d x^{2}} \psi(x)+V(x) \psi(x)=E \psi(x) .
$$

This is an ordinary second order differential equation for the stationary wave function $\psi(x)$ which may also be interpreted as an eigenvalue problem for $E$. In order to build up the notation for the supersymmetry analysis, we redefine the potential to $V_{-}(x) \equiv V(x)-E_{0}$ where $E_{0}$ is the minimal eigenvalue of (1). In this way all the energy levels get downshifted by $E_{0}$. Associated to the potential $V_{-}$we define the corresponding Hamiltonian operator

$$
H_{-} \equiv \frac{-\hbar^{2}}{2 m} \frac{d^{2}}{d x^{2}}+V_{-},
$$

for which we may write explicitly

$$
H_{-} \psi_{n}^{-}(x)=-\frac{\hbar^{2}}{2 m} \frac{d^{2}}{d x^{2}} \psi_{n}^{-}(x)+V_{-}(x) \psi_{n}^{-}(x)=E_{n}^{-} \psi_{n}^{-}(x),
$$

and where we labeled the eigenfunctions $\psi_{n}$ and eigenvalues $E_{n}$ by the index $n$. Inspired by the well-known creation and annihilation operator technique of the harmonic oscillator we assume the second order operator $H_{-}$can be factorized into

$$
H_{-}=A^{+} A^{-},
$$

with

$$
\begin{aligned}
& A^{-} \equiv \frac{\hbar}{\sqrt{2 m}} \frac{d}{d x}+W(x), \\
& A^{+} \equiv \frac{-\hbar}{\sqrt{2 m}} \frac{d}{d x}+W(x),
\end{aligned}
$$


where $W(x)$ is some real function. By substituting (5) into (4) we see this task can be accomplished if and only if $W(x)$ is a solution of the Riccati nonlinear first order differential equation

$$
V_{-}=W^{2}(x)-\frac{\hbar}{\sqrt{2 m}} W^{\prime}(x) .
$$

The quantity $W(x)$ is called the superpotential associated to the original potential $V(x)$ in (1) and satisfies the commutation relation

$$
\left[A^{-}, A^{+}\right]=\frac{2 \hbar}{\sqrt{2 m}} W^{\prime}(x) .
$$

Now switching the order between $A^{-}$and $A^{+}$in (5) we define the new operator

$$
H_{+} \equiv A^{-} A^{+}=-\frac{\hbar^{2}}{2 m} \frac{d^{2}}{d x^{2}}+V_{+},
$$

with

$$
V_{+} \equiv W^{2}(x)+\frac{\hbar}{\sqrt{2 m}} W^{\prime}(x) .
$$

Naturally, $H_{+}$may be interpreted as a new Hamiltonian related to the potential $V_{+}$. As can be easily checked, $A^{+}$and $A^{-}$are the adjoint of each other, while both Hamiltonians $H_{+}$and $H_{-}$are Hermitian semi-positive-definite operators. Denoting the eigenfunctions of $H_{+}$by $\psi_{n}^{+}$, similarly to (3) we write

$$
H_{+} \psi_{n}^{+}(x)=E_{n}^{+} \psi_{n}^{+}(x) .
$$

For simplicity, we assume $\psi_{0}^{-}(x)$ is normalizable and satisfies $A^{-} \psi_{0}^{-}=0$, which is consistent with $H_{-} \psi_{0}^{-}=0$ and leads to

$$
\psi_{0}^{-} \sim \exp \left[-\frac{\sqrt{2 m}}{\hbar} \int W(x) d x\right],
$$

with

$$
\int d x\left|\psi_{0}^{-}\right|^{2}=1
$$

On the other hand, if the ground state for $H_{+}$were to satisfy $A^{+} \psi_{0}^{+}=0$, it would be proportional to $\left(\psi_{0}^{-}\right)^{-1}$ which cannot be normalized. Therefore, considering the eigenvalues in (10) ordered by increasing value of energies, we have $E_{0}^{-}=0$ and $E_{0}^{+}>0$. This piece of assumption comprises the case conventionally known by unbroken supersymmetry $[1,2]$. 
Observing that

$$
H_{+}\left(A^{-} \psi_{n}^{-}\right)=E_{n}^{-}\left(A^{-} \psi_{n}^{-}\right),
$$

and comparing with the eigenvalue equation (10) we see that (i) the spectrum of $H_{+}$coincides with that of $H_{-}$with the sole exception of $E_{0}^{-}=0$ and (ii) the eigenfunctions of $H_{+}$are proportional to $A^{-} \psi_{n}^{-}$. Namely, the nonzero eigenvalues of $H_{ \pm}$are related by

$$
E_{n}^{+}=E_{n+1}^{-}>0, n \geq 0 .
$$

Imposing normalization to unity on all $\psi_{n}^{ \pm}(x)$ and choosing a real proportionality constant, we may write

$$
\psi_{n}^{+}=\frac{1}{\sqrt{E_{n+1}^{-}}} A^{-} \psi_{n+1}^{-}, n \geq 0 .
$$

By applying $A^{+}$to both sides of the last equation it can be inverted to

$$
\psi_{n+1}^{-}=\frac{1}{\sqrt{E_{n}^{+}}} A^{+} \psi_{n}^{+}, n \geq 0 .
$$

Note that with this notation convention we have

$$
A^{+} \psi_{0}^{+}=\sqrt{E_{0}^{+}} \psi_{1}^{-} \neq 0
$$

We see that the $A^{-}$and $A^{+}$operators connect $H_{-}$and $H_{+}$eigenstates with the same energy. Knowledge of the eigenstates and eigenvalues of one of the Hamiltonians $H_{ \pm}$leads to the knowledge of the corresponding solution for its partner.

\section{The Supersymmetry Algebra}

In the previous section, we considered $H_{-}$and $H_{+}$as two Hamiltonians governing independent problems, namely the first one characterized by the potential $V_{-}$and the second by the potential $V_{+}$. Now we construct a more general problem by absorbing these two Hamiltonians into a two-dimensional structure and where the previous solutions $\psi_{n}^{ \pm}$become related by a symmetry transformation. We begin by defining the $2 \times 2$ matrix operators

$$
Q^{+}=\left(\begin{array}{cc}
0 & A^{+} \\
0 & 0
\end{array}\right),
$$




$$
Q^{-}=\left(\begin{array}{cc}
0 & 0 \\
A^{-} & 0
\end{array}\right)
$$

as well as the full Hamiltonian operator

$$
H \equiv\left\{Q^{-}, Q^{+}\right\} \equiv Q^{-} Q^{+}+Q^{+} Q^{-}
$$

which may be written explicitly as

$$
H=\left(\begin{array}{cc}
A^{+} A^{-} & 0 \\
0 & A^{-} A^{+}
\end{array}\right)=\left(\begin{array}{cc}
H_{-} & 0 \\
0 & H_{+}
\end{array}\right) .
$$

Furthermore, we promptly note that $Q^{ \pm}$is nilpotent in the sense that

$$
\left(Q^{ \pm}\right)^{2}=0
$$

and commutes with the Hamiltonian

$$
\left[Q^{ \pm}, H\right] \equiv Q^{ \pm} H-H Q^{ \pm}=0
$$

Thus we see that the three operators $H$ and $Q^{ \pm}$constitute a basis for a faithful representation of a Lie superalgebra. To be more specific, the (anti)commutator relations (19) and (22) can be seen as a generalized graded commutator in the vector space generated by $Q^{ \pm}$and $H$. By assigning grades $\overline{0}$ to $H$ and $\overline{1}$ to $Q_{ \pm}$ we have a Lie superalgebra endowed with a $\mathbb{Z}_{2}$-graded structure.

From the physical point of view, the property $\left[Q^{ \pm}, H\right]=0$ clearly signals an underlying symmetry. Namely, the operators $Q^{ \pm}$do not change the energy of an $H$ eigenstate. This is precisely what is meant by the term supersymmetry. Let us elaborate a bit further on this point. The operators $H$ and $Q^{ \pm}$act on a Hilbert space of physical states formed by column vectors of the form

$$
\Psi(x)=\left(\begin{array}{c}
\phi_{1}(x) \\
\phi_{2}(x)
\end{array}\right)
$$

and the time-independent Schrödinger equation may be written as

$$
H \Psi=E \Psi
$$

with $H$ as in (20). Note that the two components of (24) correspond to the Schrödinger equations for the potentials $V_{-}$and $V_{+}$, and the set

$$
B=\left\{\left(\begin{array}{c}
\psi_{n}^{-}(x) \\
0
\end{array}\right),\left(\begin{array}{c}
0 \\
\psi_{n}^{+}(x)
\end{array}\right)\right\}, n=0,1, \ldots,
$$


constitutes a basis for the complete Hilbert space. By defining $E_{n} \equiv E_{n}^{-}=$ $E_{n-1}^{+}, n \geq 1$ and $E_{0} \equiv E_{0}^{-}=0$, we see the nonzero eigenvalues of (24) are doubly degenerated. Namely, we may write

$$
H \Psi_{n}=E_{n} \Psi_{n},
$$

with

$$
\Psi_{n}=\left(\begin{array}{c}
c_{-} \psi_{n}^{-} \\
c_{+} \psi_{n-1}^{+}
\end{array}\right), n \geq 1,
$$

for $c_{ \pm}$arbitrary complex numbers satisfying $\left|c_{-}\right|^{2}+\left|c_{+}\right|^{2}=1$. The ground state is nondegenerate and given by

$$
\Psi_{0}=\left(\begin{array}{c}
\psi_{0}^{-} \\
0
\end{array}\right)
$$

satisfying

$$
H \Psi_{0}=0 .
$$

Notice that $Q^{ \pm}$are generalized ladder operators in the sense that

$$
Q^{-}\left(\begin{array}{c}
\psi_{n}^{-}(x) \\
0
\end{array}\right)=\sqrt{E_{n}^{-}}\left(\begin{array}{c}
0 \\
\psi_{n-1}^{+}(x)
\end{array}\right),
$$

and

$$
Q^{+}\left(\begin{array}{c}
0 \\
\psi_{n}^{+}(x)
\end{array}\right)=\sqrt{E_{n}^{+}}\left(\begin{array}{c}
\psi_{n+1}^{-}(x) \\
0
\end{array}\right) .
$$

More generally, for $\Psi_{n}$ as in (27), we have

$$
Q^{+} \Psi_{n}=c_{+} \sqrt{E_{n}}\left(\begin{array}{c}
\psi_{n}^{-} \\
0
\end{array}\right)
$$

and

$$
Q^{-} \Psi_{n}=c_{-} \sqrt{E_{n}}\left(\begin{array}{c}
0 \\
\psi_{n-1}^{+}
\end{array}\right),
$$

which is consistent with (26) by $\left\{Q^{+}, Q^{-}\right\} \Psi_{n}=E_{n} \Psi_{n}$. Note also that the assumption of unbroken supersymmetry implies, by $A^{-} \psi_{0}^{-}=0$, that the ground state, or vacuum, of the theory satisfies both

$$
Q^{ \pm} \Psi_{0}=0 .
$$

Since $Q^{ \pm}$are not Hermitian, but rather one the Hermitian conjugate of the other, equations (32) and (33) lack a direct physical interpretation. However, we may define the Hermitian operators

$$
Q_{1}=\left(Q^{+}+Q^{-}\right)
$$




$$
Q_{2}=i\left(Q^{+}-Q^{-}\right)
$$

which may be interpreted as supersymmetric charges, and then we have

$$
Q_{1} \Psi_{n}=\sqrt{E_{n}}\left(\begin{array}{c}
c_{+} \psi_{n}^{-} \\
c_{-} \psi_{n-1}^{+}
\end{array}\right)
$$

and

$$
Q_{2} \Psi_{n}=i \sqrt{E_{n}}\left(\begin{array}{c}
c_{+} \psi_{n}^{-} \\
-c_{-} \psi_{n-1}^{+}
\end{array}\right) .
$$

We see the eigenvectors of $Q_{1}$ satisfy $c_{+}= \pm c_{-}$while those of $Q_{2}$ satisfy $c_{+}= \pm i c_{-}$, for $n \geq 1$. Now the corresponding real eigenvalues of $Q_{1}$ and $Q_{2}$ are $\pm \sqrt{E_{n}}$. For $n=0$ we have still the one dimensional eigenspace generated by (28) with null eigenvalue for both $Q_{1}$ and $Q_{2}$. In terms of the Hermitian operators, the supersymmetric algebra reads

$$
\left[H, Q_{i}\right]=0
$$

and

$$
\left\{Q_{i}, Q_{j}\right\}=2 \delta_{i j} H
$$

for $i, j=1,2$.

\section{The Supersymmetry Group}

In the previous section we have identified the algebra of SUSYQM as a Lie superalgebra generated by $H$ and $Q_{i}$. Now we show that the supersymmetry implied by relations (19) and (22), or equivalently by (39) and (40), can be realized by the action of a corresponding supergroup in the full Hilbert space of the theory. We begin by observing that the total Hamiltonian $H$ defines a symmetry group by the action of

$$
e^{i \beta H}=\exp \left[i \beta\left(\begin{array}{cc}
H_{-} & 0 \\
0 & H_{+}
\end{array}\right)\right]
$$

in the Hilbert space of states. Here $\beta$ is a real parameter. By introducing two real Grassmannian parameters $\theta_{1}$ and $\theta_{2}$ we write a general element of the full supersymmetry group as

$$
g=\exp \left[i \beta H+i \theta_{1} Q_{1}+i \theta_{2} Q_{2}\right]
$$


In order to obtain the explicit group composition law, we define

$$
\theta_{ \pm} \equiv \theta_{1} \pm i \theta_{2}
$$

and note that if

$$
g^{(k)}=\exp \left[i \beta^{(k)} H+i \theta_{1}^{(k)} Q_{1}+i \theta_{2}^{(k)} Q_{2}\right]
$$

then we have

$$
\begin{aligned}
g^{(1)} g^{(2)}= & \exp \left[i\left(\beta^{(1)}+\beta^{(2)}\right) H\right] \exp \left[i \theta_{+}^{(1)} Q^{+}+i \theta_{-}^{(1)} Q^{-}\right] \\
& \times \exp \left[i \theta_{+}^{(2)} Q^{+}+i \theta_{-}^{(2)} Q^{-}\right] .
\end{aligned}
$$

In obtaining (45) from (44), with $k=1,2$, we have used the fact that $H$ commutes with $Q_{i}$ to split out the exponentials and reorder terms. By using elementary properties of Grassmann variables and the operator identity $\left[Q^{+}, Q^{-}\right]^{2}=H^{2}$, the product of the last two exponentials in the RHS of (45) can be written as

$$
1+i\left(\theta_{+}^{(1)}+\theta_{+}^{(2)}\right) Q^{+}+i\left(\theta_{-}^{(1)}+\theta_{-}^{(2)}\right) Q^{-}+M_{(2)}+M_{(3)}+M_{(4)},
$$

with

$$
\begin{aligned}
M_{(2)}= & -\frac{1}{2}\left(\theta_{+}^{(1)} \theta_{-}^{(2)}+\theta_{-}^{(1)} \theta_{+}^{(2)}\right)\left(Q^{+} Q^{-}+Q^{-} Q^{+}\right)+ \\
& -\frac{1}{2}\left(\theta_{+}^{(1)}+\theta_{+}^{(2)}\right)\left(\theta_{-}^{(1)}+\theta_{-}^{(2)}\right)\left[Q^{+}, Q^{-}\right], \\
M_{(3)}= & -\frac{i}{2}\left(\theta_{+}^{(1)} \theta_{-}^{(2)} \theta_{+}^{(2)}+\theta_{-}^{(1)} \theta_{+}^{(2)} \theta_{+}^{(1)}\right) Q^{+} Q^{-} Q^{+}+ \\
& -\frac{i}{2}\left(\theta_{+}^{(1)} \theta_{-}^{(2)} \theta_{-}^{(1)}+\theta_{-}^{(1)} \theta_{+}^{(2)} \theta_{-}^{(2)}\right) Q^{-} Q^{+} Q^{-},
\end{aligned}
$$

and

$$
M_{(4)}=\frac{1}{4} \theta_{+}^{(1)} \theta_{-}^{(1)} \theta_{+}^{(2)} \theta_{-}^{(2)} H^{2},
$$

which then in its turn factorizes as a product

$$
\exp \left[i \theta_{+}^{(1)} Q^{+}+i \theta_{-}^{(1)} Q^{-}\right] \exp \left[i \theta_{+}^{(2)} Q^{+}+i \theta_{-}^{(2)} Q^{-}\right]=R S
$$

with

$$
R \equiv 1-\frac{1}{2}\left(\theta_{+}^{(1)} \theta_{-}^{(2)}+\theta_{-}^{(1)} \theta_{+}^{(2)}\right) H+\frac{1}{4} \theta_{+}^{(1)} \theta_{-}^{(1)} \theta_{+}^{(2)} \theta_{-}^{(2)} H^{2}
$$


and

$$
\begin{aligned}
S \equiv & 1+i\left(\theta_{+}^{(1)}+\theta_{+}^{(2)}\right) Q^{+}+i\left(\theta_{-}^{(1)}+\theta_{-}^{(2)}\right) Q^{-} \\
& -\frac{1}{2}\left(\theta_{+}^{(1)}+\theta_{+}^{(2)}\right)\left(\theta_{-}^{(1)}+\theta_{-}^{(2)}\right)\left[Q^{+}, Q^{-}\right] .
\end{aligned}
$$

Since $Q^{ \pm}$commutes with $H$, the factorization (50) substituted back in (45) leads to the closed form

$$
\begin{aligned}
g^{(1)} g^{(2)}= & \exp \left\{i\left[\beta^{(1)}+\beta^{(2)}+\frac{i}{2}\left(\theta_{+}^{(1)} \theta_{-}^{(2)}+\theta_{-}^{(1)} \theta_{+}^{(2)}\right)\right] H\right\} \\
& \times \exp \left[i\left(\theta_{+}^{(1)}+\theta_{+}^{(2)}\right) Q^{+}+i\left(\theta_{-}^{(1)}+\theta_{-}^{(2)}\right) Q^{-}\right] .
\end{aligned}
$$

Finally, back to the original variables $\theta_{i}$, we see we can write

$$
g^{(1)} g^{(2)}=g^{(3)}
$$

as defined in (44) with

$$
\begin{gathered}
\beta^{(3)}=\beta^{(1)}+\beta^{(2)}+i\left(\theta_{1}^{(1)} \theta_{1}^{(2)}+\theta_{2}^{(1)} \theta_{2}^{(2)}\right), \\
\theta_{1}^{(3)}=\theta_{1}^{(1)}+\theta_{1}^{(2)}
\end{gathered}
$$

and

$$
\theta_{2}^{(3)}=\theta_{2}^{(1)}+\theta_{2}^{(2)},
$$

which determines the group composition law. Therefore, the group defined by (42) implements the supersymmetry of SUSYQM acting in the full Hilbert space of the theory.

\section{Conclusion}

We have reviewed supersymmetry in nonrelativistic quantum mechanics by joining two partner one dimensional potentials into a full Hamiltonian enjoying supersymmetry. We have seen that the supersymmetry can be described as a transformation connecting the solutions to the two partner potentials. By assuming unbroken supersymmetry, we have identified the supersymmetric algebra of the generators as a Lie superalgebra, writing down the structure constants for the graded commutator. We have constructed explicitly the corresponding group implementing the supersymmetry in the Hilbert space. The group composition law has been obtained in terms of the exponential of either the generators $Q^{ \pm}$or $Q_{i}$, using the corresponding real Grassmann parameters $\theta_{ \pm}$or $\theta_{i}$. The group composition law has been explicitly obtained. 


\section{Acknowledgements}

The authors gratefully acknowledge insightful discussions with Professor Rafael de Lima Rodrigues.

\section{References}

[1] E. Witten, Nucl. Phys. B 188 (1981), 513-554.

[2] A. Gangopadhyaya, J. V. Mallow and C. Rasinariu, Supersymmetric Quantum Mechanics: An Introduction, World Scientific, Singapore, (2011).

[3] F. Cooper, A. Khare and U. Sukhatme, Phys. Rept. 251 (1995), 267-385.

[4] B. K. Bagchi, Supersymmetry in Quantum and Classical Mechanics, Boca Raton, USA: Chapman \& Hall (2001).

[5] R. de Lima Rodrigues, The Quantum Mechanics SUSY Algebra: An Introductory Review, CBPF-MO-003/01 (2002).

[6] R. de Lima Rodrigues, Phys. Lett. A 326 (2004), 42-46.

[7] E. S. Rodrigues, A. F. de Lima and R. de Lima Rodrigues, Dirac Equation with vector and scalar potentials via Supersymmetry in Quantum Mechanics, arXiv:1301.6148 [math-ph] (2013).

[8] D. R. Truax, V. A. Kostelecky and M. M. Nieto, J. Math. Phys. 27 (1986), 354-364.

[9] V. A. Kostelecky, D. R. Truax and M. M. Nieto, J. Math. Phys. 27 (1986), 1419-1429. 
